\title{
Connectivity Index in Vague Graphs with Application in Construction
}

\author{
Huiqin Jiang and Yongsheng Rao \\ Institute of Computing Science and Technology, Guangzhou University, Guangzhou 510006, China \\ Correspondence should be addressed to Yongsheng Rao; rysheng@gzhu.edu.cn
}

Received 13 December 2021; Accepted 10 January 2022; Published 15 February 2022

Academic Editor: Tapan Senapati

Copyright (c) 2022 Huiqin Jiang and Yongsheng Rao. This is an open access article distributed under the Creative Commons Attribution License, which permits unrestricted use, distribution, and reproduction in any medium, provided the original work is properly cited.

\begin{abstract}
The vague graph (VG), which has recently gained a place in the family of fuzzy graph (FG), has shown good capabilities in the face of problems that cannot be expressed by fuzzy graphs and interval-valued fuzzy graphs. Connectivity index (CI) in graphs is a fundamental issue in fuzzy graph theory that has wide applications in the real world. The previous definitions' limitations in the connectivity of fuzzy graphs directed us to offer new classifications in vague graph. Hence, in this paper, we investigate connectivity index, average connectivity index, and Randic index in vague graphs with several examples. Also, one of the motives of this research is to introduce some special types of vertices such as vague connectivity enhancing vertex, vague connectivity reducing vertex, and vague connectivity neutral vertex with their properties. Finally, an application of connectivity index in the selected town for building hospital is presented.
\end{abstract}

\section{Introduction}

The FG concept serves as one of the most dominant and extensively employed tools for multiple real-world problem representations, modeling, and analysis. To specify the objects and the relations between them, the graph vertices or nodes and edges or arcs are applied, respectively. Graphs have long been used to describe objects and the relationship between them. Many of the issues and phenomena around us are associated with complexities and ambiguities that make it difficult to express certainty. These difficulties were alleviated by the introduction of fuzzy sets by Zadeh [1]. This concept established well-grounded allocation membership degree to elements of a set. Actually, fuzzy set theory is one of the best and most powerful tools for modeling problems in examining the relationship between uncertainties in the real world. Rosenfeld [2] proposed the idea of FG in 1975. Kauffman [3] represented FGs based on Zadeh's fuzzy relation [4, 5]. Bhutani and Rosenfeld [6] introduced the concept of strong edges. Bhattacharya [7] presented some observations on FGs, and some operations on FGs were described by Mordeson and Peng [8]. The existence of a single degree for a true membership could not resolve the ambiguity on uncertain issues, so the need for a degree of membership was felt. Afterward, to overcome the existing ambiguities, Gau and Buehrer [9] gave false membership degrees and defined a vague set as the sum of degrees not greater than 1. Ramakrishna in [10] proposed the vague graph concept. New VG concept was introduced and analyzed by Borzooei et al. [11-13]. The vague graph, which has recently gained a place in the family of FG, has shown good capabilities in the face of problems that cannot be expressed by FGs. A vague graph is referred to as a generalized structure of an FG that conveys more exactness, adaptability, and compatibility to a system when coordinated with systems running on FGs. Furthermore, a VG is able to concentrate on determining the uncertainly coupled with the inconsistent and indeterminate information of any realworld problem, where FGs may not lead to adequate results. Connectivity index is one of the most important topics that has many applications in dynamic detection of competition condition in parallel programming, finding phylogenetic trees based on protein domain information, and social networks. The connectivity index problem can also be used 
to model many real-world situations in the fields of circuit design, telecommunications, network flow, and so on. Binu et al. [14] introduced connectivity index of a fuzzy graph. Mathew and Sunitha [15] defined several types of arc in fuzzy graph. Naeem et al. [16] investigated connectivity indices of intuitionistic fuzzy graphs (IFGs). Poulik and Ghorai [17] studied indices of graphs under bipolar fuzzy environment. Sebastian et al. [18] presented connectivity parameters in generalized fuzzy graphs. Several concepts and results in VGs were proposed and investigated by Akram et al. [19, 20]. Ghorai et al. [21] studied regular product vague graphs and product vague line graphs. References [22, 23] introduced competition-FGs and some remarks on bipolarFGs. References [24-29] investigated several concepts on FGs and VGs. Kou et al. [30] studied g-eccentric node and vague detour g-boundary nodes in VGs. Rashmanlou et al. [31-33] described categorical properties and paired domination in IFGs. Kosari et al. [34] introduced the restrained K-rainbow reinforcement number of graphs.

In this paper, we investigated connectivity index, average connectivity index, and Randic index in VGs with several examples. Likewise, we introduced some special types of nodes such as vague connectivity enhancing node, vague connectivity reducing node, and vague connectivity neutral node with their properties. Finally, an application of connectivity index in construction is presented.

\section{Preliminaries}

A FG $G=(V, v, \eta)$ is a nonempty set $V$ together with a pair of functions $v: V \longrightarrow[0,1]$ and $\eta: V \times V \longrightarrow[0,1]$ such that $\eta(a b) \leq \min \{v(a), v(b)\}$, for all $a, b \in V$. Here, $\eta$ is a symmetric fuzzy relation on $V \times V$.

Definition 1 (see [9]). A VS $M$ is a pair $\left(t_{M}, f_{M}\right)$ on set $V$ where $t_{M}$ and $f_{M}$ are taken as real valued functions which can be defined on $V \longrightarrow[0,1]$, so that $t_{M}(a)+f_{M}(a) \leq 1$, for all $a \in V$.

Definition 2 (see [10]). $G=(M, N)$ is called a VG on a crisp graph $G^{*}$, in which $M=\left(t_{M}, f_{M}\right)$ is a VS on $V$ and $N=$ $\left(t_{N}, f_{N}\right)$ is a VS on $E \subseteq V \times V$ so that $t_{N}(a b) \leq \min \left(t_{M}\right.$ $\left.(a), t_{M}(b)\right)$ and $f_{N}(a b) \geq \max \left(f_{M}(a), f_{M}(b)\right), \forall a b \in E$.

Definition 3 (see [11]). Let $G=(M, N)$ be a VG.

(i) A VG $G^{\prime}=\left(M^{\prime}, N^{\prime}\right)$ is said to be a PVSG of $G$ if $t_{M^{\prime}}(a) \leq t_{M}(a), \quad f_{M^{\prime}}(a) \geq f_{M}(a), \quad \forall a \in V \quad$ and $t_{N^{\prime}}(a b) \leq t_{N}(a b), f_{N^{\prime}}(a b) \geq f_{N}(a b)$, for each edge $a b \in G$. (ii) A VG $G^{\prime}=\left(M^{\prime}, N^{\prime}\right)$ is said to be a VSG of $G$ if $t_{M}(a)=t_{M^{\prime}}(a), \quad f_{M}(a)=f_{M^{\prime}}(a), \quad \forall a \in V^{\prime} \quad$ and $t_{N^{\prime}}(a b)=t_{N}(a b), f_{N^{\prime}}(a b)=f_{N}(a b)$, for each edge $a b \in G^{\prime}$.

Definition 4 (see [11]). Let $G=(M, N)$ be a VG.

(i) A path $p$ : $a=a_{0}, a_{1}, \ldots, a_{k-1}, a_{k}=b$ in $G$ is a sequence of distinct nodes where $t_{N}\left(a_{i-1} a_{i}\right)>0$, $f_{N}\left(a_{i-1} a_{i}\right)>0, i=1,2, \ldots, k$. The length of $P$ is $k$.

(ii) If $p: a=a_{0}, a_{1}, \ldots, a_{k-1}, a_{k}=b$ is a path between $a$ and $b$ of length $k$, then $\left(t_{N}(a b)\right)^{k}=\sup \left\{t_{N}\left(a a_{1}\right)\right.$ $\left.\wedge t_{N}\left(a_{1} a_{2}\right) \wedge \ldots \wedge t_{N}\left(a_{k-1} b\right)\right\}$ and $\left(f_{N}(a b)\right)^{k}$ $=\inf \left\{f_{N}\left(a a_{1}\right) \vee f_{N}\left(a_{1} a_{2}\right) \vee \ldots \vee f_{N}\left(a_{k-1} b\right)\right\}$.

$\operatorname{CONN}_{G}(a, b)=\left(\operatorname{CONN}_{G}^{t}(a, b), \operatorname{CONN}_{G}^{f}(a, b)\right)=\left(\left(t_{N}\right.\right.$ $\left.(a b))^{\infty},\left(f_{N}(a b)\right)^{\infty}\right)$ is called the strength of connectedness between any two nodes $a$ and $b$ in $G$ where $\operatorname{CONN}_{G}^{t}(a, b)=$ $\sup _{k \in N}\left\{\left(t_{N}(a b)\right)^{k}\right\}$ and $\operatorname{CONN}_{G}^{f}(a b)=\inf _{k \in N}\left\{\left(f_{N}(a b)\right)^{k}\right\}$. If $t_{N}(a b)>0$ and $f_{N}(a b)>0, \forall(a, b)$ in $G$, then the VG $G$ is called a CVG. If $t_{N}(a b)=\min \left\{t_{M}(a), t_{M}(b)\right\}$ and $f_{N}(a b)=\max \left\{f_{M}(a), f_{M}(b)\right\}, \forall a, b \in V$, then $G$ is named a complete VG.

Definition 5 (see [11]). For a VG $G$, if $t_{N}(a b) \geq\left(t_{N}(a b)\right)^{\infty}$ and $f_{N}(a b) \leq\left(f_{N}(a b)\right)^{\infty}$, then the arc $(a b)$ is named a strong arc of $G$.

Definition 6 (see [11]). Let $G=(M, N)$ be a CVG.

(i) $G$ is called a VT if $G$ has a VSSG $G^{\prime}=\left(A^{\prime}, B^{\prime}\right)$ which is also a tree and for all $\operatorname{arcs}(a b)$ not in $G$, $\left(t_{N^{\prime}}(a b)\right)^{\infty}<\left(t_{N}(a b)\right)^{\infty}$ and $\left(f_{N^{\prime}}(a b)\right)^{\infty}>\left(f_{N}\right.$ $(a b))^{\infty}$.

(ii) An edge $(a b)$ is said to be a VB if $\left(t_{N}(a b)\right)^{\infty}>$ $\left(t_{N^{\prime}}(a b)\right)^{\infty}$ and $\left(f_{N}(a b)\right)^{\infty}<\left(f_{N^{\prime}}(a b)\right)^{\infty}$.

(iii) $G$ is called a vague cycle if $G^{*}$ is a cycle and there does not exist unique edge $(a b)$ in $G$ for which $t_{N}(a b)=\min \left\{t_{N}\left(a_{1} b_{1}\right) \mid\left(a_{1} b_{1}\right) \in E\right\}$ and $f_{N}(a b)$ $=\max \left\{f_{N}\left(a_{1} b_{1}\right) \mid\left(a_{1} b_{1}\right) \in E\right\}$.

All the basic notations are shown in Table 1.

\section{Connectivity Index of a Vague Graph}

Definition 7. The CI of a VG $G$ denoted by $\mathrm{CI}_{\mathrm{VG}}(G)$ is defined as

$$
\begin{aligned}
\mathrm{CI}_{\mathrm{VG}}(G) & =\left(\mathrm{CI}_{\mathrm{VG}}^{t}(G), \mathrm{CI}_{\mathrm{VG}}^{f}(G)\right) \\
& =\left(\sum_{a, b \in V} t_{M}(a) t_{M}(b) \cdot \operatorname{CONN}_{G}^{t}(a, b), \sum_{a, b \in V} f_{M}(a) f_{M}(b) \cdot \operatorname{CONN}_{G}^{f}(a, b)\right),
\end{aligned}
$$


TABLE 1: Some basic notations.

\begin{tabular}{lc}
\hline Notation & Meaning \\
\hline FG & Fuzzy graph \\
VG & Vague graph \\
CVG & Connected vague graph \\
VS & Vague set \\
MV & Membership value \\
PVSG & Partial vague subgraph \\
VSG & Vague subgraph \\
VT & Vague tree \\
ACI & Average connectivity index \\
VCRN & Vague connectivity reducing node \\
VCEN & Vague connectivity enhancing node \\
VCNN & Vague connectivity neutral node \\
CEVG & Connectivity enhancing vague graph \\
CRVG & Connectivity reducing vague graph \\
NVG & Neutral vague graph \\
OND & Open neighborhood degree \\
RI & Randic index \\
CI & Connectivity index \\
\hline
\end{tabular}

where $\mathrm{CI}_{\mathrm{VG}}^{t}(G)$ and $\mathrm{CI}_{\mathrm{VG}}^{f}(G)$, respectively, denote the true$\mathrm{CI}$ and false-CI of $G$.

Example 1. Consider the CVG $G$ of the graph $G^{*}$ shown in Figure 1 , where $V=\{a, b, c, d, e\}$ and $E=\{(a, b),(b, c),(c$, $d),(d, e),(a, e),(b, d)\}$. Here, we suppose that the true-MV and false-MV of every node are 0.2 and 0.5 , respectively. Here,

$$
\begin{aligned}
& \operatorname{CONN}_{G}^{t}(a, b)=0.1, \quad \operatorname{CONN}_{G}^{t}(a, c)=0.1, \quad \operatorname{CONN}_{G}^{t}(a, d)=0.1, \\
& \operatorname{CONN}_{G}^{t}(a, e)=0.2, \quad \operatorname{CONN}_{G}^{t}(b, c)=0.2, \quad \operatorname{CONN}_{G}^{t}(b, d)=0.2, \\
& \operatorname{CONN}_{G}^{t}(b, e)=0.1, \quad \operatorname{CONN}_{G}^{t}(c, d)=0.2, \quad \operatorname{CONN}_{G}^{t}(c, e)=0.1, \\
& \operatorname{CONN}_{G}^{t}(d, e)=0.1 \text {. } \\
& \operatorname{CONN}_{G}^{f}(a, b)=0.6, \quad \operatorname{CONN}_{G}^{f}(a, c)=0.6, \quad \operatorname{CONN}_{G}^{f}(a, d)=0.6, \\
& \operatorname{CONN}_{G}^{f}(a, e)=0.6, \quad \operatorname{CONN}_{G}^{f}(b, c)=0.5, \quad \operatorname{CONN}_{G}^{f}(b, d)=0.5, \\
& \operatorname{CONN}_{G}^{f}(b, e)=0.6, \quad \operatorname{CONN}_{G}^{f}(c, d)=0.5, \quad \operatorname{CONN}_{G}^{f}(c, e)=0.6, \\
& \operatorname{CONN}_{G}^{f}(d, e)=0.6 \text {. } \\
& \mathrm{CI}_{\mathrm{VG}}^{t}(G)=0.004+0.004+0.004+0.008+0.008+0.008+0.004+0.008+0.004+0.004=0.056 \text {, } \\
& \mathrm{CI}_{\mathrm{VG}}^{f}(G)=0.15+0.15+0.15+0.15+0.125+0.125+0.15+0.125+0.15+0.15=1.425 \text {. }
\end{aligned}
$$

1.425)

Hence, $\quad \mathrm{CI}_{\mathrm{VG}}(G)=\left(\mathrm{CI}_{\mathrm{VG}}^{t}(G), \mathrm{CI}_{\mathrm{VG}}^{f}(G)\right)=(0.056$,

Theorem 1. For a PVSG $G^{\prime}=\left(M^{\prime}, N^{\prime}\right)$ of a $V G$ $G=(M, N), \quad C I_{V G}^{t}\left(G^{\prime}\right) \leq C I_{V G}^{t}(G)$ and $C I_{V G}^{f}\left(G^{\prime}\right) \geq C I_{V G}^{f}$ $(G)$.

Proof. Let $G^{\prime}=\left(M^{\prime}, N^{\prime}\right)$ be a PVSG of $G=(M, N)$. Hence, $t_{M^{\prime}}(a) \leq t_{M}(a), \quad f_{M^{\prime}}(a) \geq f_{M}(a), \quad \forall a \in G^{\prime}$ and $t_{N^{\prime}}(a b)$ $\leq t_{N}(a b), \quad f_{N^{\prime}}(a b) \geq f_{N}(a b), \quad \forall a b \in G^{\prime}$. Since $\operatorname{CONN}_{G^{\prime}}^{t}(a, b)$ and $\operatorname{CONN}_{G}^{f}(a, b)$ lie on one or many edges of $G^{\prime}$ and $\operatorname{CONN}_{G}^{t}(a, b)$ and $\operatorname{CONN}_{G}^{f}(a, b)$ lie on one or many edges of $G$ and $t_{N^{\prime}}(a b) \leq t_{N}(a b), f_{N^{\prime}}(a b) \geq f_{N}(a b)$, $\forall a b \in G^{\prime}$, and hence we have

$$
\begin{aligned}
\operatorname{CONN}_{G}^{t}(a, b) & \leq \operatorname{CONN}_{G}^{t}(a, b), \operatorname{CONN}_{G}^{f}(a, b) \\
& \geq \operatorname{CONN}_{G}^{f}(a, b), \forall(a, b) \in G^{\prime} .
\end{aligned}
$$

Since

$$
\begin{gathered}
f_{M^{\prime}}(a) f_{M^{\prime}}(b) \geq 0, \\
f_{M}(a) f_{M}(b) \geq 0 .
\end{gathered}
$$

So, 


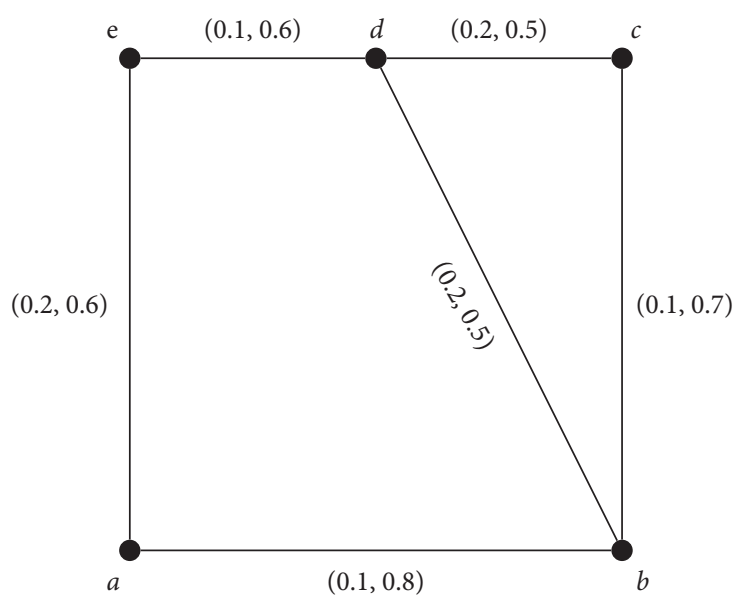

Figure 1: Connected VG G.

$t_{M^{\prime}}(a) t_{M^{\prime}}(b) \operatorname{CONN}_{G}^{t}(a, b) \leq t_{M}(a) t_{M}(b) \operatorname{CONN}_{G}^{t}(a, b)$, $f_{M^{\prime}}(a) f_{M^{\prime}}(b) \operatorname{CONN}_{G^{\prime}}^{f}(a, b) \geq f_{M}(a) f_{M}(b) \operatorname{CONN}_{G}^{f}(a, b)$.

Therefore,

$$
\begin{aligned}
& \sum_{a, b \in V^{\prime}} t_{M^{\prime}}(a) t_{M^{\prime}}(b) \operatorname{CONN}_{G}^{t}(a, b) \\
\leq & \sum_{a, b \in V} t_{M}(a) t_{M}(b) \operatorname{CONN}_{G}^{t}(a, b) \\
& \sum_{a, b \in V^{\prime}} f_{M^{\prime}}(a) f_{M^{\prime}}(b) \operatorname{CONN}_{G}^{f}(a, b) \\
\geq & \sum_{a, b \in V} f_{M}(a) f_{M}(b) \operatorname{CONN}_{G}^{f}(a, b) .
\end{aligned}
$$
$(G)$.

Therefore, $\mathrm{CI}_{\mathrm{VG}}^{t}\left(G^{\prime}\right) \leq \mathrm{CI}_{\mathrm{VG}}^{t}(G)$ and $\mathrm{CI}_{\mathrm{VG}}^{f}\left(G^{\prime}\right) \leq \mathrm{CI}_{\mathrm{VG}}^{f}$

Remark 1. For a VSG $G^{\prime}=\left(M^{\prime}, N^{\prime}\right)$ of a VG $G$, $\mathrm{CI}_{\mathrm{VG}}^{t}\left(G^{\prime}\right) \leq \mathrm{CI}_{\mathrm{VG}}^{t}(G)$ and $\mathrm{CI}_{\mathrm{VG}}^{f}\left(G^{\prime}\right) \leq \mathrm{CI}_{\mathrm{VG}}^{f}(G)$.

Example 2. Let $G^{\prime}$ be a CVSG of the VG $G$ as in Example 2 (see Figure 2). Here,

$$
\begin{aligned}
& \mathrm{CI}_{\mathrm{VG}}^{t}\left(G^{\prime}\right)=0.004+0.004+0.008+0.008+0.004+0.004=0.032 \\
& \mathrm{CI}_{\mathrm{VG}}^{f}\left(G^{\prime}\right)=0.15+0.15+0.15+0.125+0.15+0.15=0.875
\end{aligned}
$$

So, $\mathrm{CI}_{\mathrm{VG}}\left(G^{\prime}\right)=(0.032,0.875)$. Therefore, Example 2 shows that $\mathrm{CI}_{\mathrm{VG}}^{t}\left(G^{\prime}\right) \leq \mathrm{CI}_{\mathrm{VG}}^{t}(G)$ and $\mathrm{CI}_{\mathrm{VG}}^{f}\left(G^{\prime}\right) \leq \mathrm{CI}_{\mathrm{VG}}^{f}(G)$.

Theorem 2. If $G^{\prime}=\left(M^{\prime}, N^{\prime}\right)$ is a VSG of a $C V G$ $G=(M, N)$, where $V^{\prime}=V-\{s\}, \quad s \in V$, then $C I_{V G}^{t}$ $\left(G^{\prime}\right)<C I_{V G}^{t}(G)$ and $C I_{V G}^{f}\left(G^{\prime}\right)<C I_{V G}^{f}(G)$.

Proof. Let $G$ have $n$ nodes, i.e., $|V|=n$ and $s \in V$. Then, $V^{\prime}=$ $V-\{s\}$ i.e., $\left|V^{\prime}\right|=n-1$. Suppose $V=\left\{a_{1}, a_{2}, \ldots, a_{n}=s\right\}$. Hence, $V^{\prime}=\left\{a_{1}, a_{2}, \ldots, a_{n-1}\right\}$. Since $G^{\prime}$ is VSG of $G, G^{\prime}$ must be a PVSG of $G$. So,

$$
\begin{aligned}
& \mathrm{CI}_{\mathrm{VG}}^{t}(G)=\mathrm{CI}_{\mathrm{VG}}^{t}\left(G^{\prime}\right)+\sum_{i=1}^{n-1} t_{M}\left(a_{n}\right) t_{M}\left(a_{i}\right) \operatorname{CONN}_{G}^{t}\left(a_{n}, a_{i}\right), \\
& \mathrm{CI}_{\mathrm{VG}}^{f}(G)=\mathrm{CI}_{\mathrm{VG}}^{f}\left(G^{\prime}\right)+\sum_{i=1}^{n-1} f_{M}\left(a_{n}\right) f_{M}\left(a_{i}\right) \operatorname{CONN}_{G}^{f}\left(a_{n}, a_{i}\right) .
\end{aligned}
$$

Thus, $\quad \mathrm{CI}_{\mathrm{VG}}^{t}\left(G^{\prime}\right)<\mathrm{CI}_{\mathrm{VG}}^{t}(G) \quad$ and $\quad \mathrm{CI}_{\mathrm{VG}}^{f}\left(G^{\prime}\right)<$ $\mathrm{CI}_{\mathrm{VG}}^{f}(G)$.

Theorem 3. If $G$ is a complete $V G$ and $V=\left\{a_{1}, a_{2}, \ldots, a_{n}\right\}$ so that $t_{M}\left(a_{i}\right)=p_{n}$ and $f_{M}\left(a_{i}\right)=q_{i}, i=1,2, \ldots, n$, where 


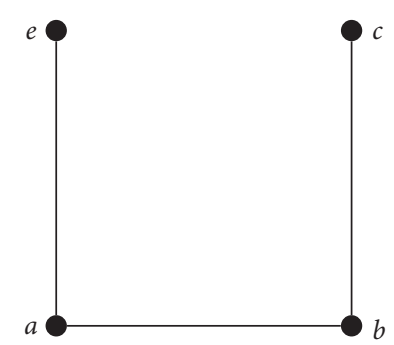

Figure 2: Connected VSG $G^{\prime}$.

$p_{1} \leq p_{2} \leq \ldots \leq p_{n}$ and $q_{1} \geq q_{2} \geq \ldots \geq q_{n}$, then $C I_{V G}^{t}(G)=$ $\sum_{i=1}^{n-1} p_{i}^{2} \sum_{j=i+1}^{n} p_{j}$ and $C I_{V G}^{f}(G)=\sum_{i=1}^{n-1} q_{i}^{2} \sum_{j=i+1}^{n} q_{j}$.

Proof. Let $a_{1} \in V, t_{M}\left(a_{1}\right)=p_{1}$, and $f_{M}\left(a_{1}\right)=q_{1}$. Since $G$ is complete, $\operatorname{CONN}_{G}^{t}\left(a_{i}, a_{j}\right)=t_{N}\left(a_{i}, a_{j}\right)$ and $\operatorname{CONN}_{G}^{f}\left(a_{i}\right.$, $\left.a_{j}\right)=f_{N}\left(a_{i}, a_{j}\right)$. Then, $\operatorname{CONN}_{G}^{t}\left(a_{1}, a_{j}\right)=t_{M}\left(a_{1}\right)=p_{1}$, $\operatorname{CONN}_{G}^{f}\left(a_{1}, a_{j}\right)=f_{M}\left(a_{1}\right)=q_{1}, j>1$. Since $p_{1} \leq p_{2} \leq \ldots$ $\leq p_{n}$ and $q_{1} \geq q_{2} \geq \ldots \geq q_{n}$, then $t_{M}\left(a_{1}\right) \leq t_{M}\left(a_{2}\right) \leq \ldots$ $\leq t_{M}\left(a_{n}\right)$ and $f_{M}\left(a_{1}\right) \geq f_{M}\left(a_{2}\right) \geq \ldots \geq f_{M}\left(a_{n}\right)$. Therefore,

$$
t_{M}\left(a_{1}\right) t_{M}\left(a_{j}\right) \operatorname{CONN}_{G}^{t}\left(a_{1} a_{j}\right)=p_{1} p_{j} p_{1}=p_{1}^{2} p_{j},
$$$$
f_{M}\left(a_{1}\right) f_{M}\left(a_{j}\right) \operatorname{CONN}_{G}^{f}\left(a_{1} a_{j}\right)=q_{1} q_{j} q_{1}=q_{1}^{2} q_{j}
$$

It shows that

$$
\begin{gathered}
\sum_{j=2}^{n} t_{M}\left(a_{1}\right) t_{M}\left(a_{j}\right) \operatorname{CONN}_{G}^{t}\left(a_{1}, a_{j}\right)=\sum_{j=2}^{n} p_{1}^{2} p_{j}, \\
\sum_{j=2}^{n} f_{M}\left(a_{1}\right) f_{M}\left(a_{j}\right) \operatorname{CONN}_{G}^{f}\left(a_{1}, a_{j}\right)=\sum_{j=2}^{n} q_{1}^{2} q_{j} .
\end{gathered}
$$

Therefore,

$$
\begin{aligned}
\sum_{i=1}^{n-1} \sum_{j=2}^{n} t_{M}\left(a_{i}\right) t_{M}\left(a_{j}\right) \operatorname{CONN}_{G}^{t}\left(a_{i}, a_{j}\right) & =\sum_{i=1}^{n-1} \sum_{j=2}^{n} p_{i}^{2} p_{j}, \\
\sum_{i=1}^{n-1} \sum_{j=2}^{n} f_{M}\left(a_{i}\right) f_{M}\left(a_{j}\right) \operatorname{CONN}_{G}^{f}\left(a_{i}, a_{j}\right) & =\sum_{i=1}^{n-1} \sum_{j=2}^{n} q_{i}^{2} q_{j} .
\end{aligned}
$$

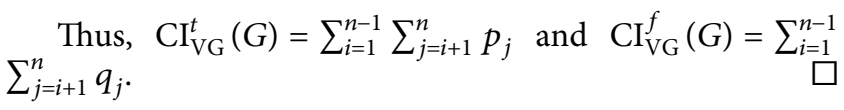

Example 3. Consider the complete VG $G$ shown in Figure 3 where $V=\{a, b, c\}$. Here, $t_{M}\left(v_{i}\right)=p_{i}$ and $f_{M}\left(v_{i}\right)=q_{i}$, for $i=1,2,3$.

$$
t_{M}(a)=p_{1}=0.1, f_{M}(a)=q_{1}=0.4, t_{M}(b)=p_{2}=0.2,
$$
$f_{M}(b)=q_{2}=0.3, t_{M}(c)=p_{3}=0.3, f_{M}(c)=q_{3}=0.2$.

Hence, $p_{1} \leq p_{2} \leq p_{3}$ and $q_{1} \geq q_{2} \geq q_{3}$.

$$
\begin{aligned}
\mathrm{CI}_{\mathrm{VG}}^{t}(G) & =\sum_{i=1}^{2} p_{i}^{2} \sum_{j=i+1}^{3} p_{j} \\
& =p_{1}^{2} p_{2}+p_{1}^{2} p_{3}+p_{2}^{2} p_{3} \\
& =(0.1)^{2}(0.2)+(0.1)^{2}(0.3)+(0.2)^{2}(0.3)=0.017 \\
\mathrm{CI}_{\mathrm{VG}}^{f}(G) & =\sum_{i=1}^{2} q_{i}^{2} \sum_{j=i+1}^{3} q_{j} \\
& =q_{1}^{2} q_{2}+q_{1}^{2} q_{3}+q_{2}^{2} q_{3} \\
& =0.048+0.032+0.018=0.098 .
\end{aligned}
$$

Thus, $\mathrm{CI}_{\mathrm{VG}}(G)=\left(\mathrm{CI}_{\mathrm{VG}}^{t}(G), \mathrm{CI}_{\mathrm{VG}}^{f}(G)\right)=(0.017,0.098)$. Now we define average connectivity index (ACI) of a VG.

Definition 8. The ACI of a VG $G=(M, N)$ denoted by $\mathrm{ACI}_{\mathrm{VG}}(G)$ is defined as

$$
\begin{aligned}
\operatorname{ACI}_{\mathrm{VG}}(G) & =\left(\operatorname{ACI}_{\mathrm{VG}}^{t}(G), \operatorname{ACI}_{\mathrm{VG}}^{f}(G)\right) \\
& =\frac{1}{\left(\begin{array}{l}
n \\
2
\end{array}\right)}\left[\sum_{a, b \in V} t_{M}(a) t_{M}(b) \operatorname{CONN}_{G}^{t}(a, b), \sum_{a, b \in V} f_{M}(a) f_{M}(b) \operatorname{CONN}_{G}^{f}(a, b)\right] .
\end{aligned}
$$

Example 4. From Example 2, for VG G, the ACI of $G$ is 


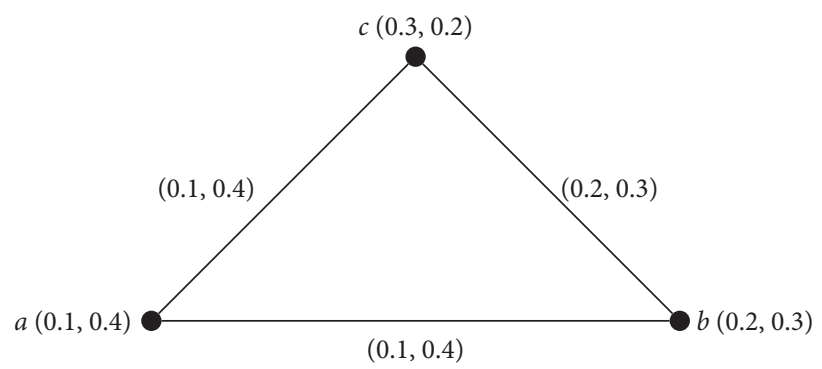

Figure 3: A complete VG G.

$$
\begin{aligned}
\operatorname{ACI}_{\mathrm{VG}}(G) & =\left(\operatorname{ACI}_{\mathrm{VG}}^{t}(G), \operatorname{ACI}_{\mathrm{VG}}^{f}(G)\right)=\left(\frac{0.056}{\left(\begin{array}{c}
5 \\
2
\end{array}\right)}, \frac{1.425}{\left(\begin{array}{c}
5 \\
2
\end{array}\right)}\right) \\
& =\left(\frac{0.056}{10}, \frac{1.425}{10}\right)=(0.0056,0.1425) .
\end{aligned}
$$

(i) VCRN if $\mathrm{AC}_{\mathrm{VG}}^{t}(G-\{a\})<\mathrm{AC}_{\mathrm{VG}}^{t}(G)$ and $\mathrm{ACI}_{\mathrm{VG}}^{f}$ $(G-\{a\})>\operatorname{ACI}_{\mathrm{VG}}^{f}(G)$.

(ii) $\mathrm{VCEN}$ if $\mathrm{ACI}_{\mathrm{VG}}^{t}(G-\{a\})>\mathrm{ACI}_{\mathrm{VG}}^{t}(G)$ and $\mathrm{ACI}_{\mathrm{VG}}^{f}$ $(G-\{a\})<\operatorname{ACI}_{\mathrm{VG}}^{f}(G)$.

(iii) $\mathrm{VCNN}$ if $\mathrm{ACI}_{\mathrm{VG}}^{t}(G-\{a\})=\mathrm{ACI}_{\mathrm{VG}}^{t}(G)$ and $\mathrm{ACI}_{\mathrm{VG}}^{f}$ $(G-\{a\})=\operatorname{ACI}_{\mathrm{VG}}^{f}(G)$.

Example 5. Consider the VG G shown in Figure 4. We have

Definition 9. Let $G=(M, N)$ be a CVG. A node $a$ is called a

$$
\begin{aligned}
& \operatorname{CONN}_{G}^{t}(a, b)=0.1, \operatorname{CONN}_{G}^{t}(a, c)=0.1, \operatorname{CONN}_{G}^{t}(a, d)=0.1, \\
& \operatorname{CONN}_{G}^{t}(b, c)=0.2, \operatorname{CONN}_{G}^{t}(b, d)=0.2, \operatorname{CONN}_{G}^{t}(c, d)=0.2, \\
& \operatorname{CONN}_{G}^{f}(a, b)=0.4, \operatorname{CONN}_{G}^{f}(a, c)=0.5, \operatorname{CONN}_{G}^{f}(a, d)=0.4, \\
& \operatorname{CONN}_{G}^{f}(b, c)=0.5, \operatorname{CONN}_{G}^{f}(b, d)=0.4, \operatorname{CONN}_{G}^{f}(c, d)=0.5 .
\end{aligned}
$$

Hence,

$$
\begin{aligned}
& \mathrm{CI}_{\mathrm{VG}}^{t}(G)=0.004+0.004+0.004+0.008+0.008+0.008=0.036 \text {, } \\
& \mathrm{CI}_{\mathrm{VG}}^{f}(G)=0.036+0.045+0.045+0.036+0.045+0.036+0.045=0.243 . \\
& \mathrm{ACI}_{\mathrm{VG}}^{t}(G)=\frac{0.036}{6}=0.006, \quad \mathrm{ACI}_{\mathrm{VG}}^{f}(G)=0.040, \\
& \mathrm{ACI}_{\mathrm{VG}}^{t}(G-\{a\})=0.008, \quad \mathrm{ACI}_{\mathrm{VG}}^{f}(G-\{a\})=0.039, \\
& \mathrm{ACI}_{\mathrm{VG}}^{t}(G-\{b\})=0.005, \quad \mathrm{ACI}_{\mathrm{VG}}^{f}(G-\{b\})=0.042, \\
& \mathrm{ACI}_{\mathrm{VG}}^{t}(G-\{c\})=0.005, \quad \mathrm{ACI}_{\mathrm{VG}}^{f}(G-\{c\})=0.036, \\
& \mathrm{ACI}_{\mathrm{VG}}^{t}(G-\{d\})=0.005, \quad \mathrm{ACI}_{\mathrm{VG}}^{f}(G-\{d\})=0.042
\end{aligned}
$$

Hence, $a$ is a VCEN of $G$ and $b$ and $d$ are VCRN of $G$. 


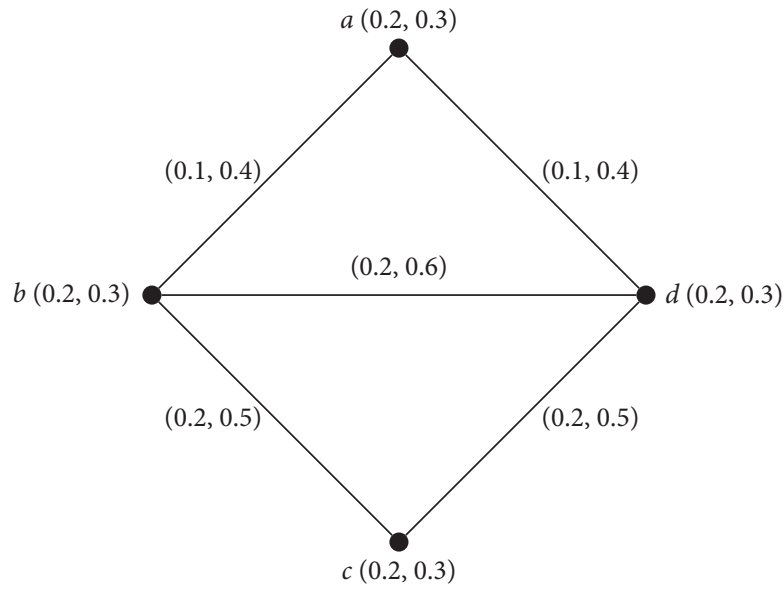

Figure 4: A connected VG G.

Theorem 4. Let $G=(M, N)$ be a complete $V G$ with $|V|=n \geq 3$, and $p_{1}=C I_{V G}^{t}(G) / C I_{V G}^{f}(G-\{a\}), \quad p_{2}=C I_{V G}^{f}$ $(G) / C I_{V G}^{f}(G-\{a\}), a \in V$. The node $a$ is $a$

(i) VCEN if and only if $p_{1} \leq n / n-2$ and $p_{2}>n / n-2$.

(ii) VCRN if and only if $p_{1}>n / n-2$ and $p_{2}<n / n-2$.

(iii) VCNN if and only if $p_{1}=n / n-2$ and $p_{2}=n / n-2$.

Proof. (i) Assume that $a$ is a VCEN. Then, Definition 9 shows that

$$
\begin{aligned}
& \operatorname{ACI}_{\mathrm{VG}}^{t}(G)<\operatorname{ACI}_{\mathrm{VG}}^{t}(G-\{a\}), \\
& \mathrm{ACI}_{\mathrm{VG}}^{f}(G)>\mathrm{ACI}_{\mathrm{VG}}^{f}(G-\{a\}) .
\end{aligned}
$$

So, $\mathrm{CI}_{\mathrm{VG}}^{t}(G) /(n / 2)<\mathrm{CI}_{\mathrm{VG}}^{t}(G-\{a\}) /(n-1 / 2)$ and $\mathrm{CI}_{\mathrm{VG}}^{f}$ $(G) /(n / 2)<\mathrm{CI}_{\mathrm{VG}}^{f}(G-\{a\}) /(n-1 / 2)$. Hence, $\mathrm{CI}_{\mathrm{VG}}^{t}(G) /$ $\mathrm{CI}_{\mathrm{VG}}^{t}(G-\{a\})<(n / 2) /(n-1 / 2)$ and $\mathrm{CI}_{\mathrm{VG}}^{f}(G) / \mathrm{CI}_{\mathrm{VG}}^{f G}(G-$ $\{a\})>(n / 2) /(n-1 / 2)$. Thus, $p_{1}<n / n-2$ and $p_{2}>n / n-2$.

Conversely, let $p_{1}<n / n-2$ and $p_{2}>n / n-2$. So, $\mathrm{CI}_{\mathrm{VG}}^{t}(G) / \mathrm{CI}_{\mathrm{VG}}^{t}(G-\{a\})<n / n-2$ and $\mathrm{CI}_{\mathrm{VG}}^{f}(G) / \mathrm{CI}_{\mathrm{VG}}^{f}(G-$ $\{a\})>n / n-2$. Hence, $\mathrm{CI}_{\mathrm{VG}}^{t}(G) /(n / 2)<\mathrm{CI}_{\mathrm{VG}}^{t}(G-\{a\}) /(n-$ $1 / 2)$ and $\mathrm{CI}_{\mathrm{VG}}^{f}(G) /(n / 2)>\mathrm{CI}_{\mathrm{VG}}^{f}(G-\{a\}) /(n-1 / 2)$. Thus, $\operatorname{ACI}_{\mathrm{VG}}^{t}(G)<\mathrm{ACI}_{\mathrm{VG}}^{t}(G-\{a\}) \quad$ and $\operatorname{ACI}_{\mathrm{VG}}^{f}(G)>\mathrm{ACI}_{\mathrm{VG}}^{f}$ $(G-\{a\})$. Therefore, $a$ is a VCEN of $G$. In the same way, (ii) and (iii) can be proved.
Theorem 5. Let $G=(M, N)$ be a complete $V G$ with $|V|=n \geq 3$. Suppose $L^{t}=\sum_{a \in V-\{b\}} t_{M}(a) t_{M}(b) \operatorname{CONN}_{G}^{t}(a, b)$, $L^{f}=\sum_{a \in V-\{b\}} f_{M}(a) f_{M}(b) \operatorname{CONN}_{G}^{f}(a, b)$, and $b$ is an end node of $G$. Then,

(i) $L^{t}<2 / n-2$ and $L^{f}>2 / 2-n$ iff $b$ is a VCEN.

(ii) $L^{t}>2 / n-2$ and $L^{f}<2 / 2-n$ iff $b$ is a VCRN.

(iii) $L^{t}=2 / n-2$ and $L^{f}=2 / 2-n$ iff $b$ is a VCNN.

Proof. (i) Let $L^{t}<2 / 2-n$ and $L^{f}>2 / 2-n$. Now,

$\mathrm{CI}_{\mathrm{VG}}^{t}(G)=\mathrm{CI}_{\mathrm{VG}}^{t}(G-\{b\})+\sum_{a \in V\{b\}} t_{M}(a) t_{M}(b) \operatorname{CONN}_{G}^{t}(a, b)$,

$\mathrm{CI}_{\mathrm{VG}}^{f}(G)=\mathrm{CI}_{\mathrm{VG}}^{f}(G-\{b\})+\sum_{a \in V\{b\}} f_{M}(a) f_{M}(b) \operatorname{CONN}_{G}^{f}(a, b)$.

So, $\quad \mathrm{CI}_{\mathrm{VG}}^{t}(G)=\mathrm{CI}_{\mathrm{VG}}^{t}(G-\{b\})+L^{t}$ and $\quad \mathrm{CI}_{\mathrm{VG}}^{f}(G)=$ $\mathrm{CI}_{\mathrm{VG}}^{f}(G-\{b\})+L^{f}$. Hence, $\mathrm{CI}_{\mathrm{VG}}^{t}(G) /(n / 2)=\mathrm{CI}_{\mathrm{VG}}^{t}(G-$ $\{a\}) /(n / 2)+L^{t} /(n / 2)$ and $\mathrm{CI}_{\mathrm{VG}}^{f}(G) /(n / 2)=\mathrm{CI}_{\mathrm{VG}}^{f}(G-$ $\{a\}) /(n / 2)+L^{f} /(n / 2)$. Thus, $\mathrm{ACI}_{\mathrm{VG}}^{t}(G)<\mathrm{CI}_{\mathrm{VG}}^{t}(G-\{b\}) /$ $(n-1 / 2) \cdot(n-2) / n+2 / n-2 /(n / 2)$ and $\mathrm{ACI}_{\mathrm{VG}}^{f}(G)>\mathrm{CI}_{\mathrm{VG}}^{f}$ $(G-\{b\}) /(n-1 / 2) \cdot(n-2) / n+2 / 2-n /(n / 2)$. Therefore,

$$
\begin{aligned}
& \operatorname{ACI}_{\mathrm{VG}}^{t}(G)<\operatorname{ACI}_{\mathrm{VG}}^{t}(G-\{b\})-\frac{2}{n}\left[\operatorname{ACI}_{\mathrm{VG}}^{t}(G-\{b\})-\frac{2}{(n-1)(n-2)}\right], \\
& \operatorname{ACI}_{\mathrm{VG}}^{f}(G)>\operatorname{ACI}_{\mathrm{VG}}^{f}(G-\{b\})-\frac{2}{n}\left[\operatorname{ACI}_{\mathrm{VG}}^{f}(G-\{b\})+\frac{2}{(n-1)(n-2)}\right] .
\end{aligned}
$$


So, $\quad \mathrm{ACI}_{\mathrm{VG}}^{t}(G)<\mathrm{ACI}_{\mathrm{VG}}^{t}(G-\{b\})$ and $\quad \mathrm{ACI}_{\mathrm{VG}}^{f}(G)>$ $\mathrm{ACI}_{\mathrm{VG}}^{f}(G-\{b\})$.

In the same way, (ii) and (iii) can be proved.

Definition 10. Let $G=(M, N)$ be a CVG.

(i) $G$ is named CEVG, if it has at least one VCEN.

(ii) $G$ is named CRVG, if it has no VCRN.

(iii) $G$ is named NVG, if all nodes of $G$ are VCNN.
Example 6. For the VG $G$ in Example 5, the node $a$ is a VCEN of $G$, so $G$ is a CEVG, but $G$ is neither a CRVG nor a NVG.

Theorem 6. For each pair of numbers $\left(L_{1}, L_{2}\right)$, where $L_{1}$ is a positive true real number and $L_{2}$ is a positive false real number, there always exist $V G G=(M, N)$ so that $C I_{V G}^{t}(G)=L_{1}$ and $C I_{V G}^{f}(G)=L_{2},|V|=n$.

Proof. Suppose $t_{M}(a)=1, \forall a \in V$, and $f_{M}(a)=0, \forall a \in V$. Now, we consider a path in $G$ so that $t_{N}(a b)=L_{1} /(n / 2)$ and $f_{N}(a b)=L_{2} /(n / 2)$ for all edges $(a, b) \in G$. Hence, we get

$$
\begin{aligned}
& \mathrm{CI}_{\mathrm{VG}}^{t}(G)=\sum_{a, b \in V} t_{M}(a) t_{M}(b) \operatorname{CONN}_{G}^{t}(a, b)=\left(\begin{array}{l}
n \\
2
\end{array}\right) \frac{L_{1}}{\left(\begin{array}{l}
n \\
2
\end{array}\right)}=L_{1}, \\
& \mathrm{CI}_{\mathrm{VG}}^{f}(G)=\sum_{a, b \in V} f_{M}(a) f_{M}(b) \operatorname{CONN}_{G}^{f}(a, b)=\left(\begin{array}{l}
n \\
2
\end{array}\right) \frac{L_{2}}{\left(\begin{array}{l}
n \\
2
\end{array}\right)}=L_{2} .
\end{aligned}
$$

Definition 11. The OND or degree of a node $c$ in a VG $G$ is described as $\operatorname{deg}(a)=\left(\operatorname{deg}^{t}(c), \operatorname{deg}^{f}(c)\right)$, where $\operatorname{deg}^{t}(c)=$ $\sum_{c \neq d} t_{N}(c d)$ and $\operatorname{deg}^{f}(c)=\sum_{c \neq d} f_{N}(c d)$. If $c d \in E \quad c d \in E$ $\operatorname{deg}(c)=\left(t_{1}, t_{2}\right), \forall c \in V$, then $G$ is named $\left(t_{1}, t_{2}\right)$-regular.
Now, we introduce the RI of a VG with examples.

Definition 12. The RI of a VG $G=(M, N)$ is shown by $\mathrm{RI}_{\mathrm{VG}}(G)$ and described as

$$
\begin{aligned}
\mathrm{RI}_{\mathrm{VG}}(G) & =\left(\mathrm{RI}_{\mathrm{VG}}^{t}(G), \mathrm{RI}_{\mathrm{VG}}^{f}(G)\right) \\
& =\left(\sum_{\substack{i \neq j \\
a_{i} a_{j} \in E}}\left(t_{M}\left(a_{i}\right) t_{M}\left(a_{j}\right) \operatorname{deg}^{t}\left(a_{i}\right) \operatorname{deg}^{t}\left(a_{j}\right)\right)^{-1 / 2}, \sum_{\substack{i \neq j \\
a_{i} a_{j} \in E}}\left(f_{M}\left(a_{i}\right) f_{M}\left(a_{j}\right) \operatorname{deg}^{f}\left(a_{i}\right) \operatorname{deg}^{f}\left(a_{j}\right)\right)^{-1 / 2}\right) .
\end{aligned}
$$

Example 7. Consider the VG $G$ shown in Figure 5. Here, $\operatorname{deg}(a)=(0.3,1.3), \operatorname{deg}(b)=(0.6,1.8), \operatorname{deg}(c)=(0.4,1.5)$, $\operatorname{deg}(d)=(0.4,1.7)$, and $\operatorname{deg}(e)=(0.3,1.1)$. 


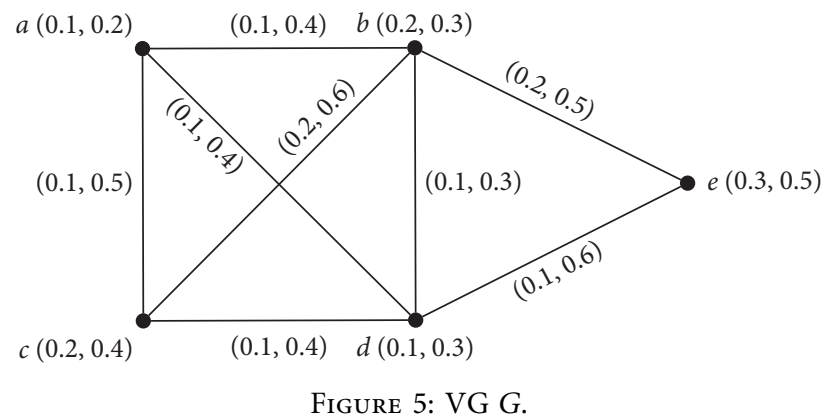

$$
\begin{aligned}
& \sum_{\substack{i \neq j \\
v_{i} v_{j} \in E}}\left(t_{M}\left(v_{i}\right) t_{M}\left(v_{j}\right) \operatorname{deg}^{t}\left(v_{i}\right) \operatorname{deg}^{t}\left(v_{j}\right)\right)^{-1 / 2} \\
= & 16.667+20.449+28.901+10.214 \\
& +17.699+14.450+9.624+16.667 \\
= & 134.671,
\end{aligned}
$$$$
\sum_{i \neq j}\left(f_{M}\left(v_{i}\right) f_{M}\left(v_{j}\right) \operatorname{deg}^{f}\left(v_{i}\right) \operatorname{deg}^{f}\left(v_{j}\right)\right)^{-1 / 2}
$$$$
v_{i} v_{j} \in E
$$$$
=2.669+2.532+2.746+1.756
$$$$
+1.807+1.905+1.835+1.889
$$$$
=17.139 \text {. }
$$

Therefore, $\mathrm{RI}_{\mathrm{VG}}(G)=\left(\mathrm{RI}_{\mathrm{VG}}^{t}(G), \mathrm{RI}_{\mathrm{VG}}^{f}(G)\right)=(134.671$, 17.139).

Theorem 7. Let $G=(M, N)$ be a connected $V G$ and $G^{\prime}=$ $\left(M^{\prime}, N^{\prime}\right)$ so that $V^{\prime}=V-\left\{c_{n}\right\}, c_{n} \in V$ with $|V|=n$. Then, $R I_{V G}^{t}(G) \geq R I_{V G}^{t}\left(G^{\prime}\right)$ and $R I_{V G}^{f^{\prime}}(G) \geq R I_{V G}^{f}\left(G^{\prime}\right)$.

Proof. $G$ has $n$ nodes, i.e., $V=\left\{c_{1}, c_{2}, \ldots, c_{n}\right\}$. Then, $V^{\prime}=\left\{c_{1}, c_{2}, \ldots, c_{n-1}\right\}$. So, $V^{\prime}$ should be a subset of $V$, so $G^{\prime}$ is a VSG of $G$. Hence, $t_{M}\left(c_{i}\right)=t_{M^{\prime}}\left(c_{i}\right), f_{M}\left(c_{i}\right)=f_{M^{\prime}}\left(c_{i}\right)$, $t_{N}\left(c_{i} c_{j}\right)=t_{N^{\prime}}\left(c_{i} c_{j}\right)$, and $f_{N}\left(c_{i} c_{j}\right)=f_{N^{\prime}}\left(c_{i} c_{j}\right), \forall c_{i} \in V^{\prime}$ and $\forall c_{i} c_{j} \in E^{\prime}$.

Now, $\operatorname{deg}^{t}\left(c_{i}\right)=\sum_{c_{i} c_{j} \in E_{j}} c_{j} t_{N}\left(c_{i} c_{j}\right)$ and $\operatorname{deg}^{f}\left(c_{i}\right)=$ $\sum_{c_{i} c_{j} \in E^{c_{j}}} f_{N}\left(c_{i} c_{j}\right)$, i.e., $\operatorname{deg}^{t}\left(c_{i}\right)$ and $\operatorname{deg}^{f}\left(c_{i}\right)$ are sum of the true-MVs and false-MVs of the edges incident in $c_{i}$ in $G$, respectively. Then, $\operatorname{deg}^{f}\left(c_{i}\right) \operatorname{deg}^{f}\left(c_{j}\right)$ is $s$ positive real number. So, $\operatorname{deg}^{\prime t}\left(c_{i}\right)=\sum_{c_{i} c_{j} \in E^{\prime}} c_{j} t_{N}\left(c_{i} c_{j}\right)$ and $\operatorname{deg}^{\prime f}$ $\left(c_{i}\right)=\sum_{c_{i} c_{j} \in E^{\prime}} c_{j} f_{N}\left(c_{i} c_{j}\right)$, i.e., $\operatorname{deg}^{\prime t}\left(c_{i}\right)$ and $\operatorname{deg}^{\prime f}\left(c_{i}\right)$ are sum of the T-MVs and false-MVs of the edges incident in $c_{i}$ in $G^{\prime}$, respectively. Then, $\operatorname{deg}^{\prime f}\left(c_{i}\right) \operatorname{deg}^{\prime f}\left(c_{j}\right)$ is a positive real number. Thus,

$$
\begin{gathered}
\left(t_{M}\left(c_{i}\right) t_{M}\left(c_{j}\right) \operatorname{deg}^{t}\left(c_{i}\right) \operatorname{deg}^{t}\left(c_{j}\right)\right)^{-1 / 2} \geq 0, \\
\left(t_{M^{\prime}}\left(c_{i}\right) t_{M^{\prime}}\left(c_{j}\right) \operatorname{deg}^{\prime t}\left(c_{i}\right) \operatorname{deg}^{\prime t}\left(c_{j}\right)\right)^{-1 / 2} \geq 0, \\
\left(f_{M}\left(c_{i}\right) f_{M}\left(c_{j}\right) \operatorname{deg}^{f}\left(c_{i}\right) \operatorname{deg}^{f}\left(c_{j}\right)\right)^{-1 / 2} \geq 0, \\
\left(f_{M^{\prime}}\left(c_{i}\right) f_{M^{\prime}}\left(c_{j}\right) \operatorname{deg}^{\prime f}\left(c_{i}\right) \operatorname{deg}^{\prime}\left(c_{j}\right)\right)^{-1 / 2} \geq 0 .
\end{gathered}
$$

So,

$$
\begin{aligned}
& \sum_{\substack{1 \leq i \neq j \leq n \\
c_{i} c_{j} \in E}}\left(t_{M}\left(c_{i}\right) t_{M}\left(c_{j}\right) \operatorname{deg}_{M}^{t}\left(c_{i}\right) \operatorname{deg}_{M}^{t}\left(c_{j}\right)\right)^{-1 / 2} \\
\geq & \sum_{\substack{1 \leq i \neq j \leq n-1 \\
c_{i} c_{j} \in E^{\prime}}}\left(t_{M^{\prime}}\left(c_{i}\right) t_{M^{\prime}}\left(c_{j}\right) \operatorname{deg}^{\prime t}\left(c_{i}\right) \operatorname{deg}^{\prime t}\left(c_{j}\right)\right)^{-1 / 2} \\
& \sum_{\substack{1 \leq i \neq j \leq n \\
c_{i} c_{j} \in E}}\left(f_{M}\left(c_{i}\right) f_{M}\left(c_{j}\right) \operatorname{deg}_{M}^{f}\left(c_{i}\right) \operatorname{deg}_{M}^{f}\left(c_{j}\right)\right)^{-1 / 2} \\
\geq & \sum_{\substack{1 \leq i \neq j \leq n-1 \\
c_{i} c_{j} \in E^{\prime}}}\left(f_{M^{\prime}}\left(c_{i}\right) f_{M^{\prime}}\left(c_{j}\right) \operatorname{deg}^{\prime f}\left(c_{i}\right) \operatorname{deg}^{\prime f}\left(c_{j}\right)\right)^{-1 / 2} .
\end{aligned}
$$
$\mathrm{RI}_{\mathrm{VG}}^{f}\left(G^{\prime}\right)$.

Example 8. Consider VG $G^{\prime}=\left(M^{\prime}, N^{\prime}\right)$ of Figure 6 and VG $G$ of Figure 5. Clearly, $t_{M}\left(v_{i}\right) \geq t_{M^{\prime}}\left(v_{i}\right), f_{M}\left(v_{i}\right) \leq f_{M^{\prime}}\left(v_{i}\right)$, $\forall v_{i} \in V^{\prime}$, and $t_{N}\left(v_{i} v_{j}\right) \geq t_{N^{\prime}}\left(v_{i} v_{j}\right), \quad f_{N}\left(v_{i} v_{j}\right) \leq f_{N^{\prime}}\left(v_{i} v_{j}\right)$, $\forall v_{i} v_{j} \in E^{\prime}$. Hence, $G^{\prime}$ is a VSG of the VG G. 


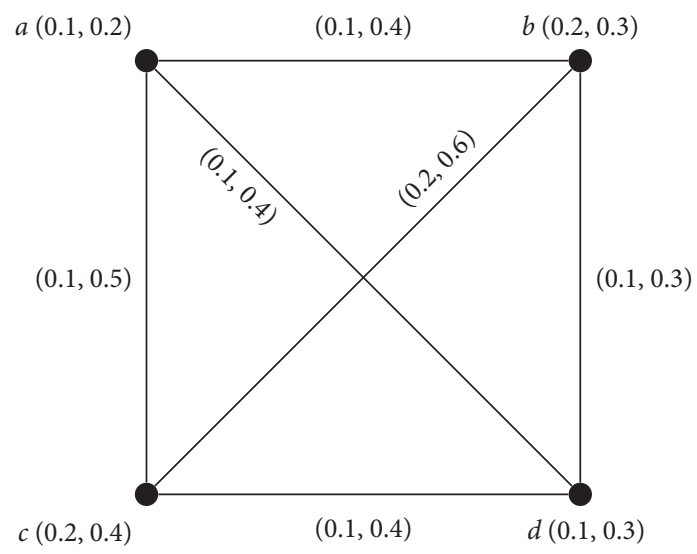

Figure 6: Vague subgraph $G^{\prime}$ of the vague graph $G$ of Figure 5.

$$
\begin{aligned}
\mathrm{RI}_{\mathrm{VG}}^{t}\left(G^{\prime}\right) & =20.449+20.449+33.333+12.5+20.449+20.449 \\
& =127.629, \\
\mathrm{RI}_{\mathrm{VG}}^{f}\left(G^{\prime}\right) & =3.140+2.532+3.414+2.067+2.247+2.788 \\
& =16.188 .
\end{aligned}
$$

Therefore, $\mathrm{RI}_{\mathrm{VG}}^{t}(G) \geq \mathrm{RI}_{\mathrm{VG}}^{t}\left(G^{\prime}\right)$ and $\mathrm{RI}_{\mathrm{VG}}^{f}(G) \geq \mathrm{RI}_{\mathrm{VG}}^{f}\left(G^{\prime}\right)$.

Remark 2. Let $G=(M, N)$ be a connected $\mathrm{VG}$ and $G^{\prime}=$ $\left(M^{\prime}, N^{\prime}\right) \quad$ so that $E^{\prime}=E-\left\{c_{i} c_{j}\right\}, \quad c_{i} c_{j} \in E$. Then, $\mathrm{RI}_{\mathrm{VG}}^{t}(G) \geq \mathrm{RI}_{\mathrm{VG}}^{t}\left(G^{\prime}\right)$ and $\mathrm{RI}_{\mathrm{VG}}^{f}(G) \geq \mathrm{RI}_{\mathrm{VG}}^{f}\left(G^{\prime}\right)$.

Proof. Assume $G$ has $n$ nodes, i.e., $|V|=n$ and $c_{i} \in V$. Let $V=\left\{c_{1}, c_{2}, \ldots, c_{n}\right\}$. So, $V^{\prime}=\left\{c_{1}, c_{2}, \ldots c_{i-1}, c_{i+1}, \ldots, c_{n}\right\}$. Then, $V^{\prime}$ must be a subset of $V$, and so $G^{\prime}$ is a $V G S$ of $G$. So, $\mathrm{RI}_{\mathrm{VG}}^{t}(G) \geq \mathrm{RI}_{\mathrm{VG}}^{t}\left(G^{\prime}\right)$ and $\mathrm{RI}_{\mathrm{VG}}^{f}(G) \geq \mathrm{RI}_{\mathrm{VG}}^{f}\left(G^{\prime}\right)$.

Theorem 8. Let $G$ be a complete $V G$ so that $M$ is a constant function. Then, $R I_{V G}(G)=\left(n / 2 v_{1}^{2}, n / 2 v_{2}^{2}\right)$, where $n=|V|$ and $\left(\nu_{1}, v_{2}\right)=\left(t_{M}\left(c_{i}\right), f_{M}\left(c_{i}\right)\right), c_{i} \in V$.

Proof. Since $M$ is constant and $t_{M}\left(c_{i}\right)=v_{1}, f_{M}\left(c_{i}\right)=v_{2}$, $c_{i} \in V$, then $t_{M}\left(c_{i}\right)=v_{1}, f_{M}\left(c_{i}\right)=v_{2}, \forall c_{i} \in V$. Since $G$ is a complete VG, $t_{N}\left(c_{i} c_{j}\right)=\min \left\{t_{M}\left(c_{i}\right), t_{M}\left(c_{j}\right)\right\}=v_{1} \quad$ and $f_{N}\left(c_{i} c_{j}\right)=\max \left\{f_{M}\left(c_{i}\right), f_{M}\left(c_{j}\right)\right\}=v_{2}, \forall c_{i} c_{j} \in E$. Again, $G$ is complete and $|V|=n$, and thus there are $n(n-1) / 2$ nodes and $n(n-1) / 2$ edges and each node in $G$ is neighbor to $(n-1)$ nodes. Therefore,

$$
\begin{aligned}
\operatorname{deg}^{t}\left(c_{i}\right) & =\sum_{\substack{c_{j} \\
c_{i} c_{j} \in E}} t_{N}\left(c_{i} c_{j}\right)=v_{1} v_{1} \ldots v_{1}=(n-1) \nu_{1}, \\
\operatorname{deg}^{f}\left(c_{i}\right) & =\sum_{c_{j}} f_{N}\left(c_{i} c_{j}\right)=v_{2} v_{2} \ldots v_{2}=(n-1) v_{2} .
\end{aligned}
$$

Hence,

$$
\begin{aligned}
\mathrm{RI}_{\mathrm{VG}}^{t}(G) & =\sum_{i \neq j}\left(t_{M}\left(c_{i}\right) t_{M}\left(c_{j}\right) \operatorname{deg}^{t}\left(c_{i}\right) \operatorname{deg}^{t}\left(c_{j}\right)\right)^{-1 / 2} \\
& =\frac{n(n-1)}{2}\left(v_{i} v_{1} \in E\right. \\
\mathrm{RI}_{\mathrm{VG}}^{f}(G) & =\sum_{i \neq j}\left(f_{M}\left(c_{i}\right) f_{M}\left(c_{j}\right) \operatorname{deg}^{f}\left(c_{i}\right) \operatorname{deg}^{f}\left(c_{j}\right)\right)^{-1 / 2} \\
& =\frac{\left.n(n-1) \nu_{1}\right)^{-1 / 2}=\frac{n}{2 v_{1}^{2}},}{2}\left(v_{2} \nu_{2}(n-1) v_{2}(n-1) v_{2}\right)^{-1 / 2}=\frac{n}{2 v_{2}^{2}} .
\end{aligned}
$$
$\left.n / 2 v_{2}^{2}\right)$.

Therefore, $\quad \mathrm{RI}_{\mathrm{VG}}(G)=\left(\mathrm{RI}_{\mathrm{VG}}^{t}(G), \mathrm{RI}_{\mathrm{VG}}^{f}(G)\right)=\left(n / 2 v_{1}^{2}\right.$,

Example 9. Let $G$ be a VG as shown in Figure 7. Here, $V=\{a, b, c, d, e\}, \quad n=|V|=5, \quad$ and $\quad t_{M}\left(v_{i}\right)=0.3=v_{1}$, $f_{M}\left(v_{i}\right)=0.5=v_{2}, 1 \leq i \leq 5$. Hence, $M$ is a constant function.

$$
E=\{a b, a c, a d, a e, b c, b d, b e, c d, c e, d e\}
$$

and $t_{N}\left(v_{i} v_{j}\right)=0.3, f_{N}\left(v_{i} v_{j}\right)=0.5$, for all edges $v_{i} v_{j} \in E$. Now, $t_{N}\left(v_{i} v_{j}\right)=0.3=\min \left\{t_{M}\left(v_{i}\right), t_{M}\left(v_{j}\right)\right\}$ and $f_{N}\left(v_{i}\right.$ 


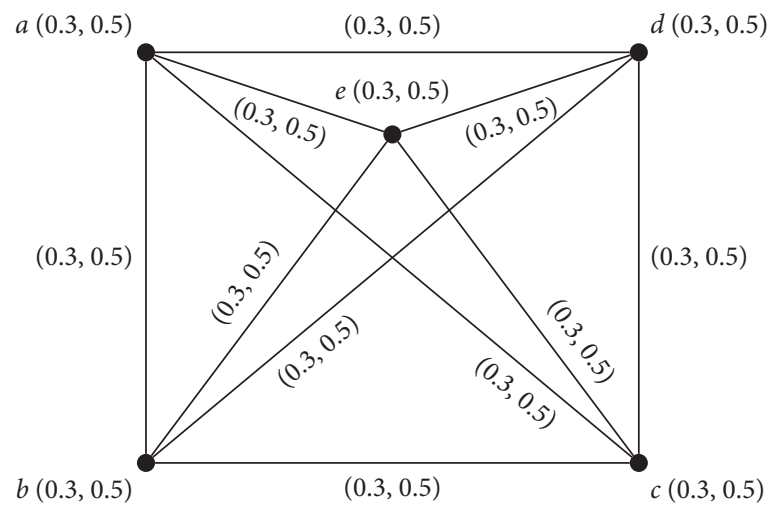

Figure 7: A VG G.

$\left.v_{j}\right)=0.5=\max \left\{f_{M}\left(v_{i}\right), f_{M}\left(v_{j}\right)\right\}$. So, $G$ is a complete VG. Using Theorem 8,

$$
\begin{aligned}
& \mathrm{RI}_{\mathrm{VG}}^{t}(G)=\frac{n}{2 v_{1}^{2}}=\frac{5}{2(0.3)^{2}}=27.77 \\
& \mathrm{RI}_{\mathrm{VG}}^{f}(G)=\frac{n}{2 v_{2}^{2}}=\frac{5}{2(0.5)^{2}}=10 .
\end{aligned}
$$

\section{Connectivity Index in the Selected Town for Building Hospital}

Today, the treatment and medical services issue sits among the most important issues for every country. Governments are always trying to provide the best possible services and medical facilities for patients. In the past, unfortunately, as a result of the lack of medical services and the lack of hospitals and clinics, patients were not transferred to these medical centers for the required time and so they lost their lives. This crisis was because of the governments' constraints for revenue and financial problems. So, governments decided to build hospitals in the cities so that patients could seek treatment without stress as soon as possible. But determining the right place to build a hospital is very important because firstly, it must be built in a place where there is no traffic and crowds nearby, and secondly, patients living in neighboring cities can easily access it. Therefore, in this paper, by using the concept of connectivity index, we try to determine the most appropriate places to establish a hospital. For this purpose, we consider six cities in China (Guangdong state), namely, Shaoguan, Qingyuan, Heyuan, Zhaoqing, Foshan, and Guangzhou, and the attributing symbols for each city are given in the graph as $A, B, C, D, E$, and $F$, respectively. Location of cities is shown in Figure 8. Also, distance between cities is shown in Table 2 .

In this vague graph, the nodes representing cities and edges also represent the quality of roads as well as the amount of traffic of cars during most hours of the day. Weight of nodes and edges is shown in Tables 3 and 4 .

The vertex $B(0.6,0.3)$ shows that the city of Qingyuan has $60 \%$ of the necessary facilities and equipment to build a hospital, of which the necessary manpower is also part of this equipment. Clearly, it does not have $30 \%$ of the equipments for construction. The $\mathrm{AB}$ edge shows that the Shaoguan-Qingyuan route has $50 \%$ quality and the necessary road and transportation standards (road quality and traffic signs), and the amount of car traffic on this route is equal to $50 \%$. For the vague graph $G$ shown in Figure 9, we have 


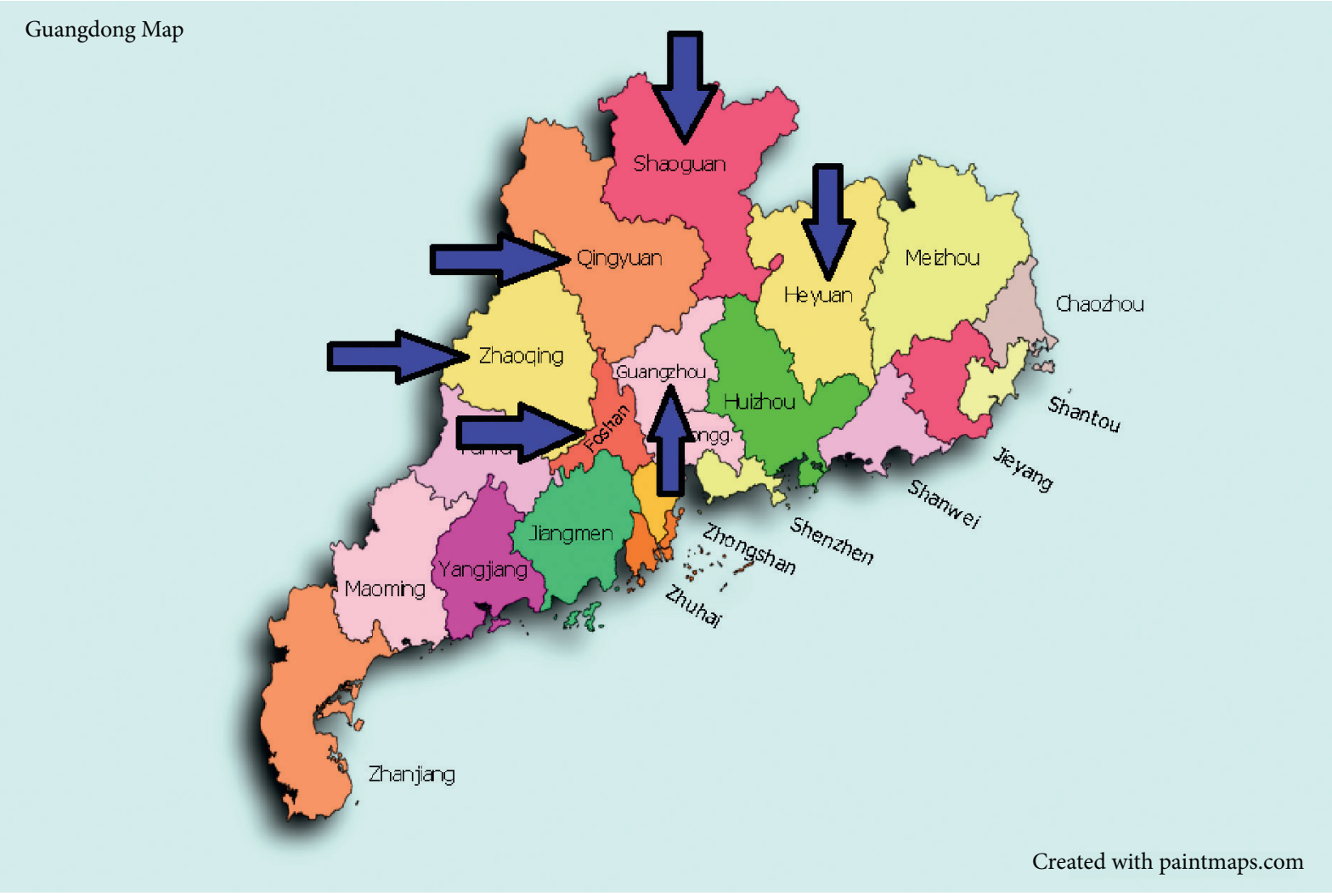

Figure 8: Location of cities.

TABle 2: Distance between cities.

\begin{tabular}{lcc}
\hline Cities & Shaoguan-Foshan & Foshan-Zhaoqing \\
Distance & $202 \mathrm{~km}$ & $69 \mathrm{~km}$ \\
Cities & Shaoguan-Zhaoqing & Shaoguan-Qingyuan \\
Distance & $225 \mathrm{~km}$ & $134 \mathrm{~km}$ \\
Cities & Qingyuan-Heyuan & Heyuan-Zhaoqing \\
Distance & $168 \mathrm{~km}$ & $293 \mathrm{~km}$ \\
Cities & Zhaoqing-Guangzhou & \\
Distance & $81 \mathrm{~km}$ & \\
\hline
\end{tabular}

TABle 3: Weight of nodes in $G$.

\begin{tabular}{lcccccc}
\hline$G$ & $A$ & $B$ & $C$ & $D$ & $F$ & $E$ \\
$\left(t_{M}, f_{M}\right)$ & $(0.5,0.3)$ & $(0.6,0.2)$ & $(0.5,0.4)$ & $(0.3,0.5)$ & $(0.6,0.3)$ & $(0.7,0.2)$ \\
\hline
\end{tabular}

TABle 4: Weight of edges in G.

\begin{tabular}{lccc}
\hline$G$ & $A E$ & $A D$ & $A B$ \\
$\left(t_{N}, f_{N}\right)$ & $(0.4,0.5)$ & $(0.2,0.6)$ & $(0.5,0.5)$ \\
$G$ & $E D$ & $D F$ & $B C$ \\
$\left(t_{N}, f_{N}\right)$ & $(0.2,0.6)$ & $(0.3,0.6)$ & $(0.5,0.4)$ \\
$G$ & $C D$ & & \\
$\left(t_{N}, f_{N}\right)$ & $(0.2,0.5)$ & & \\
\hline
\end{tabular}




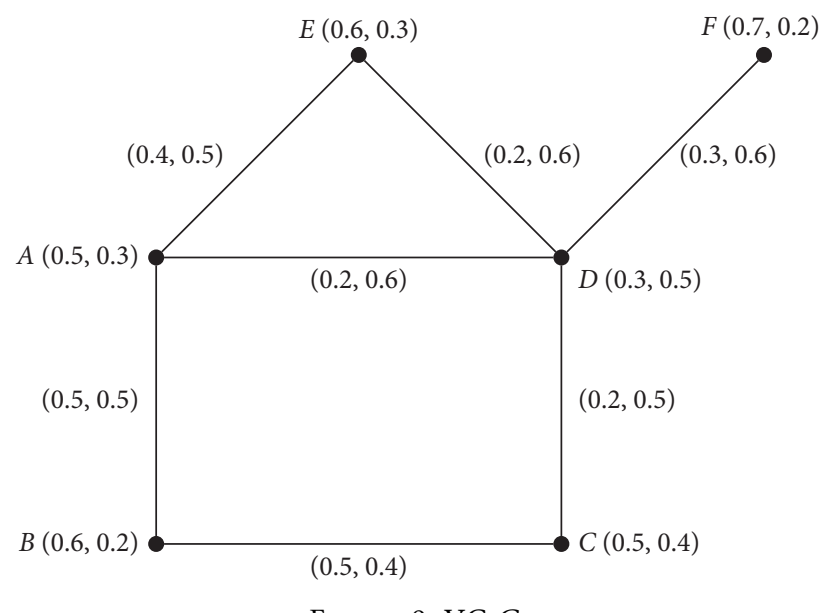

Figure 9: VG G.

$$
\begin{aligned}
& \operatorname{CONN}_{G}^{t}(A, E)=0.4, \quad \operatorname{CONN}_{G}^{t}(A, D)=0.2, \quad \operatorname{CONN}_{G}^{t}(A, F)=0.2, \\
& \operatorname{CONN}_{G}^{t}(A, B)=0.5, \quad \operatorname{CONN}_{G}^{t}(A, C)=0.5, \quad \operatorname{CONN}_{G}^{t}(B, E)=0.4, \\
& \operatorname{CONN}_{G}^{t}(B, D)=0.2, \quad \operatorname{CONN}_{G}^{t}(B, C)=0.5, \quad \operatorname{CONN}_{G}^{t}(B, F)=0.2 \text {, } \\
& \operatorname{CONN}_{G}^{t}(C, D)=0.2, \quad \operatorname{CONN}_{G}^{t}(C, E)=0.4, \quad \operatorname{CONN}_{G}^{t}(C, F)=0.2 \text {, } \\
& \operatorname{CONN}_{G}^{t}(D, E)=0.2, \quad \operatorname{CONN}_{G}^{t}(D, F)=0.3, \quad \operatorname{CONN}_{G}^{t}(E, F)=0.2 \text {, } \\
& \operatorname{CONN}_{G}^{f}(A, E)=0.5, \quad \operatorname{CONN}_{G}^{f}(A, D)=0.5, \quad \operatorname{CONN}_{G}^{f}(A, F)=0.6, \\
& \operatorname{CONN}_{G}^{f}(A, B)=0.5, \quad \operatorname{CONN}_{G}^{f}(A, C)=0.5, \quad \operatorname{CONN}_{G}^{f}(B, E)=0.5, \\
& \operatorname{CONN}_{G}^{f}(B, D)=0.5, \quad \operatorname{CONN}_{G}^{f}(B, C)=0.4, \quad \operatorname{CONN}_{G}^{f}(B, F)=0.6, \\
& \operatorname{CONN}_{G}^{f}(C, D)=0.5, \quad \operatorname{CONN}_{G}^{f}(C, E)=0.5, \quad \operatorname{CONN}_{G}^{f}(C, F)=0.6, \\
& \operatorname{CONN}_{G}^{f}(D, E)=0.5, \quad \operatorname{CONN}_{G}^{f}(D, F)=0.6, \quad \operatorname{CONN}_{G}^{f}(E, F)=0.6, \\
& \mathrm{CI}_{\mathrm{VG}}^{t}(G)=0.12+0.03+0.07+0.15+0.125+0.144+0.036+0.15+0.084+0.03+0.12+0.07+0.036+0.063+0.084 \\
& =1.312 \text {, } \\
& \mathrm{CI}_{\mathrm{VG}}^{f}(G)=0.045+0.075+0.036+0.03+0.06+0.03+0.05+0.032+0.024+0.1+0.06+0.048+0.075+0.06+0.036 \\
& =0.761 \text {. } \\
& \mathrm{ACI}_{\mathrm{VG}}^{t}(G)=\frac{1.312}{15}=0.0874
\end{aligned}
$$$$
\mathrm{ACI}_{\mathrm{VG}}^{f}(G)=\frac{0.761}{15}=0.0507 .
$$

In the same way, we have 


$$
\begin{aligned}
\mathrm{CI}_{\mathrm{VG}}^{t}(G-\{A\})=1.817, & \mathrm{CI}_{\mathrm{VG}}^{f}(G-\{A\})=0.515, \\
\mathrm{CI}_{\mathrm{VG}}^{t}(G-\{B\})=0.748, & \mathrm{CI}_{\mathrm{VG}}^{f}(G-\{B\})=0.595, \\
\mathrm{CI}_{\mathrm{VG}}^{t}(G-\{C\})=0.817, & \mathrm{CI}_{\mathrm{VG}}^{f}(G-\{C\})=0.461, \\
\mathrm{CI}_{\mathrm{VG}}^{t}(G-\{D\})=1.117, & \mathrm{CI}_{\mathrm{VG}}^{f}(G-\{D\})=0.401, \\
\mathrm{CI}_{\mathrm{VG}}^{t}(G-\{E\})=0.808, & \mathrm{CI}_{\mathrm{VG}}^{f}(G-\{E\})=0.515, \\
\mathrm{CI}_{\mathrm{VG}}^{t}(G-\{F\})=0.941, & \mathrm{CI}_{\mathrm{VG}}^{f}(G-\{F\})=0.557 . \\
\mathrm{ACI}_{\mathrm{VG}}^{t}(G-\{A\})=0.1817, & \mathrm{ACI}_{\mathrm{VG}}^{t}(G-\{B\})=0.0748, \\
\mathrm{ACI}_{\mathrm{VG}}^{t}(G-\{C\})=0.0817, & \mathrm{ACI}_{\mathrm{VG}}^{t}(G-\{D\})=0.1117, \\
\mathrm{ACI}_{\mathrm{VG}}^{t}(G-\{E\})=0.0808, & \mathrm{ACI}_{\mathrm{VG}}^{t}(G-\{F\})=0.0941, \\
\mathrm{ACI}_{\mathrm{VG}}^{f}(G-\{A\})=0.0515, & \mathrm{ACI}_{\mathrm{VG}}^{f}(G-\{B\})=0.0595, \\
\mathrm{ACI}_{\mathrm{VG}}^{f}(G-\{C\})=0.0461, & \mathrm{ACI}_{\mathrm{VG}}^{f}(G-\{D\})=0.0401, \\
\mathrm{ACI}_{\mathrm{VG}}^{f}(G-\{E\})=0.0515, & \mathrm{ACI} I_{\mathrm{VG}}^{f}(G-\{F\})=0.0557 .
\end{aligned}
$$

Hence,

$$
\begin{array}{ll}
\operatorname{ACI}_{\mathrm{VG}}^{t}(G-\{B\})<\operatorname{ACI}_{\mathrm{VG}}^{t}(G) & \operatorname{ACI}_{\mathrm{VG}}^{f}(G-\{B\})>\operatorname{ACI}_{\mathrm{VG}}^{f}(G), \\
\mathrm{ACI}_{\mathrm{VG}}^{t}(G-\{D\})>\operatorname{ACI}_{\mathrm{VG}}^{t}(G) & \mathrm{ACI}_{\mathrm{VG}}^{f}(G-\{D\})<\operatorname{ACI}_{\mathrm{VG}}^{f}(G), \\
\operatorname{ACI}_{\mathrm{VG}}^{t}(G-\{E\})<\operatorname{ACI}_{\mathrm{VG}}^{t}(G) & \mathrm{ACI}_{\mathrm{VG}}^{f}(G-\{E\})>\operatorname{ACI}_{\mathrm{VG}}^{f}(G) .
\end{array}
$$

Clearly, the vertices $B$ and $E$ are VCRN and the vertex $D$ is VCEN. If we remove the node $D$, then the true-CI is strictly increased and false-CI is strictly decreased. Also, by deleting vertex $D$, vertex $F$ is automatically deleted (because it is completely disconnected).

Therefore, if the government wants to build a hospital in one of these six cities for the treatment of patients, it should avoid the cities of Zhaoqing and Guangzhou. Also, the cities of Qingyuan and Foshan are the best choices for building a hospital because firstly, they have the most facilities and human resources for construction, and secondly, the communication roads between other cities with these two cities are of better quality and have the least traffic and congestion compared to other routes.

\section{Conclusion}

The vague graphs can amplify flexibility and precision to model complex real-time problems better than a fuzzy graph. They have several applications in many decisionmaking processes among solution choice, weather forecasting, prognosis risks in business, and so on, and one of the most important features of VGs that has many applications in real problems is the concept of connectivity index. Connectivity index has many applications in psychology, medical sciences, social groups, and computer networks. Therefore, in this paper, we examined connectivity index, average connectivity index, and Randic index in VGs with

several examples. Likewise, some special types of nodes such as vague connectivity enhancing vertex, vague connectivity reducing vertex, and vague connectivity neutral vertex are presented. Finally, an application of connectivity index in construction has been introduced. In our future work, we will define the domination of the VGs in terms of strong edges and examine their properties. Also, we will study domination in terms of independent sets, and since many of the phenomena surrounding us are hybrid, we also discuss the domination concept on its fuzzy operations.

\section{Data Availability}

No data were used to support this study.

\section{Conflicts of Interest}

The authors declare that they have no conflicts of interest.

\section{Acknowledgments}

This work was supported by the National Key R\&D Program of China (No. 2018YFB1005100), the National Natural Science Foundation of China (No. 62172116), and the Guangzhou Academician and Expert Workstation (No. 20200115-9). 


\section{References}

[1] L. A. Zadeh, "Fuzzy sets," Information and Control, vol. 8, no. 3, pp. 338-353, 1965.

[2] A. Rosenfeld, Fuzzy Graphs, Fuzzy Sets and Their Applications, L. A. Zadeh, K. S. Fu, and M. Shimura, Eds., Academic Press, New York, NY, USA, 1975.

[3] A. Kauffman, "Introduction a la theories des sous-emsembles 503 ous," Masson Cie, vol. 1, pp. 607-618, 1973.

[4] L. A. Zadeh, "Similarity relations and fuzzy orderings," Information Sciences, vol. 3, no. 2, pp. 177-200, 1971.

[5] L. A. Zadeh, "Is there a need for fuzzy logic?" Information Sciences, vol. 178, no. 13, pp. 2751-2779, 2008.

[6] K. R. Bhutani and A. Rosenfeld, "Strong arcs in fuzzy graphs," Information Sciences, vol. 152, pp. 319-322, 2003.

[7] P. Bhattacharya, "Some remarks on fuzzy graphs," Pattern Recognition Letters, vol. 6, no. 5, pp. 297-302, 1987.

[8] J. N. Mordeson and C.-S. Peng, "Operations on fuzzy graphs," Information Sciences, vol. 79, no. 3-4, pp. 159-170, 1994.

[9] W.-L. Gau and D. J. Buehrer, "Vague sets," IEEE Transactions on Systems, Man, and Cybernetics, vol. 23, no. 2, pp. 610-614, 1993.

[10] N. Ramakrishna, "Vague graphs," International Journal of Cognitive Computing, vol. 7, pp. 51-58, 2009.

[11] R. Borzooei and H. Rashmanlou, "Domination in vague graphs and its applications," Journal of Intelligent and Fuzzy Systems, vol. 29, no. 5, pp. 1933-1940, 2015.

[12] R. A. Borzooei and H. Rashmanlou, "Degree of vertices in vague graphs," Journal of applied mathematics \& informatics, vol. 33, no. 5, pp. 545-557, 2015.

[13] S. Banitalebi, R. A. Borzooei, and E. Mohammaszadeh, "2Domination in vague graphs," Algebraic Structures and their Applications, vol. 8, no. 2, pp. 203-222, 2021.

[14] M. Binu, S. Mathew, and J. N. Mordeson, "Connectivity index of a fuzzy graph and its application to human traffcking," Fuzzy Sets and Systems, vol. 360, 2018.

[15] S. Mathew and M. S. Sunitha, "Types of arcs in a fuzzy graph," Information Sciences, vol. 179, no. 11, pp. 1760-1768, 2009.

[16] T. Naeem, A. Gumaei, M. Kamran Jamil, A. Alsanad, and K. Ullah, "Connectivity indices of intuitionistic fuzzy graphs and their applications in internet routing and transport network flow," Mathematical Problems in Engineering, vol. 2021, Article ID 4156879, 16 pages, 2021.

[17] S. Poulik and G. Ghorai, "Certain indices of graphs under bipolar fuzzy environment with Applications," Soft Computing, vol. 24, pp. 1-13, 2020.

[18] A. Sebastian, J. N. Mordeson, and S. Mathew, "Generalized fuzzy graph connectivity parameters with application to human trafficking," Mathematics, vol. 8, no. 3, p. 424, 2020.

[19] M. Akram, W. A. Dudek, and M. Murtaza Yousof, "Regularity in vague intersection graphs and vague line graphs," Abstract and Applied Analysis, vol. 2014, Article ID 525389, 10 pages, 2014.

[20] M. Akram, A. N. Gani, and A. B. Saeid, "Vague hypergraphs," Journal of Intelligent and Fuzzy Systems, vol. 26, no. 2, pp. 647-653, 2014.

[21] G. Ghorai, M. Pal, and C. H. Li, "Regular product vague graphs and product vague line graphs," Cogent Mathematics, vol. 3, no. 1, Article ID 1213214, 2016.

[22] S. Samanta and M. Pal, "Fuzzy k-competition graphs and p-competition fuzzy graphs," Fuzzy Information and Engineering, vol. 5, no. 2, pp. 191-204, 2013.
[23] S. Samanta and M. Pal, "Irregular bipolar fuzzy graphs," International Journal of Fuzzy System Applications, vol. 2, pp. 91-102, 2012.

[24] M. Dettlaff, M. Lemanska, S. Kosari, and S. Sheikholeslami, "The convex domination subdivision number of a graph," Communications in Combinatorics and Optimization, vol. 1, pp. 43-56, 2016.

[25] S. Kosari, Y. Rao, H. Jiang, X. Liu, P. Wu, and Z. Shao, "Vague graph structure with application in medical diagnosis," Symmetry, vol. 12, no. 10, p. 1582, 2020.

[26] Y. Rao, S. Kosari, and Z. Shao, "Certain Properties of vague graphs with a novel application," Mathematics, vol. 8, no. 10, p. 1647, 2020.

[27] Y. Rao, S. Kosari, Z. Shao, R. Cai, and L. Xinyue, “A Study on Domination in vague incidence graph and its application in medical sciences," Symmetry, vol. 12, no. 11, p. 1885, 2020.

[28] Z. Shao, S. Kosari, H. Rashmanlou, and M. Shoaib, "New concepts in intuitionistic fuzzy graph with application in water supplier systems," Mathematics, vol. 8, no. 8, p. 1241, 2020.

[29] X. Shi and S. Kosari, "Certain properties of domination in product vague graphs with an application in medicine," Frontiers in Physics, vol. 9, pp. 1-8, Article ID 680634, 2021.

[30] Z. Kou, S. Kosari, and M. Akhoundi, "A novel description on vague graph with application in transportation systems," Journal of Mathematics, vol. 2021, Article ID 4800499, 11 pages, 2021.

[31] H. Rashmanlou, S. Samanta, M. Pal, and R. A. Borzooei, "Intuitionistic fuzzy graphs with categorical properties," Fuzzy Information and Engineering, vol. 7, no. 3, pp. 317-334, 2015.

[32] H. Rashmanlou and R. A. Borzooei, "A note on vague graphs," Algebraic Structures and Their Applications, vol. 2, no. 1, pp. 9-19, 2015.

[33] S. Sahoo, M. Pal, H. Rashmanlou, and R. A. Borzooei, "Covering and paired domination in intuitionistic fuzzy graphs," Journal of Intelligent and Fuzzy Systems, vol. 33, no. 6, pp. 4007-4015, 2017.

[34] S. Shaebani, S. Kosari, and L. Asgharsharghi, "The restrained K-rainbow reinforcement number of graphs," Discrete Mathematics, Algorithms and Applications, vol. 13, no. 3, Article ID 2150026, 2021. 